\title{
Conformational Dynamics of Heme-pocket in Myoglobin Encapsulated in Silica Sol-gel Glasses
}

\author{
Seongheun Kim, Taegon Lee, and Manho Lim \\ Department of Chemistry and Chemistry Institute for Functional Haterials, Pusan National Lniversitv, Busan 609-735, Korea \\ E-mail: mhimapusanackr \\ Received November 5,2006
}

Key Words : Conformational relaxation of mỵoglobin. Heme pocket. Femtosecond IR spectroscopy. Silica sol-gel. Spatial confinement

Local and global conformational motions of protein are essential to ligand transport and binding and thus protein function. ${ }^{1}$ Various environmental factors influence the kinetics and/or thermodynamics of structural transitions. Spatial confinement of protein can alter the conformational dynanics and thus influence its activity ${ }^{2,3}$ It is of great interest to understand protein functionality in the confuned physiological environment where it often operates. ${ }^{+}$The influence of spatial confinement of protein dynamics, structure, and function can be studied by encapsulating protein in silica sol-gel glasses. ${ }^{-4}$ Nanoporous networks with interpore connections in silica sol-gel glasses allow transport of small molecules into and out of the glasses at reasonable rates but retaining the protein molecules within their pores. ${ }^{3.3}$ The mesoporosity and framework rigidity of sol-gel glasses has been shown to stabilize entrapped protein structure against temperature. $\mathrm{pH}$, and chemical denaturation. ${ }^{3,5}$ Tertiary and quaternary changes in heme proteins were found to be drantatically slowed and geninate recombination of $\mathrm{CO}$ photodissociated from these proteins are greatly enhanced. ${ }^{6}$

Ligand binding in myoglobin (Mb) has long served as a biological model reaction. ${ }^{7}$ Fentosecond IR experiments of photolyzed carbonmonoxy nyoglobin $(\mathrm{MbCO})$ at anbient temperature showed that $\mathrm{CO}$ becomes temporarily trapped in a nearby ligand docking site before escaping into the surrounding solvent. ${ }^{8}$ The printary docking site (PDS), denoted $B$ state. is on top of the heme group about $2 \AA$ from the active binding site. It has been suggested that the PDS serves as a station mediating the passage of ligand to and from the active binding site. thereby nodulating ligand-binding activity. ${ }^{8}$ Fentosecond IR spectroscopy has shown two distinct stretch bands for $\mathrm{CO}$ in the $B$ state of $\mathrm{Mb}$. ${ }^{9}$ Those bands, denoted $B_{1}$ and $B_{2}$, arise from two opposite orientations of CO. Recently we have shown that conformational dynamics of the heme pocket PDS can be probed by time-resolved vibrational spectra of $\mathrm{CO}$ photodissociated from $\mathrm{MbCO}{ }^{11.11}$ We have suggested that the spectral evolution is induced by protein conformational changes that influence structure and electric field of the heme pocket. ${ }^{10.11}$ We have found that the magnitude of the slow rate in the spectral shift of $B_{1}$ band decreases with increasing solvent viscosity and suggested that it serve as a reporter of global conformational change of heme proteins after deligation. ${ }^{[i, 1]}$ Here we have extended our early investigations to $\mathrm{MbCO}$ encapsulated in sol-gel glasses to characterize conformational dynamics of heme pocket of $\mathrm{Mb}$ in confined environment. Global confonmational dynamics of $\mathrm{Mb}$ entrapped in sol-gel glasses were deduced from the spectral evolution of $B$ bands

As shown in Figure I(a), equilibrium absorption spectrum of the stretching mode of $\mathrm{CO}$ in $\mathrm{MbCO}$ encapsulated in solgel glasses is similar to that in $\mathrm{D}_{2} \mathrm{O}$. However. main band in sol-gel is shifted to slightly higher frequency and the $A_{0}$ band at $1920 \mathrm{~cm}^{-1}$ has larger portion in the sample of sol-gel. Solvent is known to modify the resonance frequencies of the vibrational bands and the partitioning between different taxonomic structural substates of the proteins. The similarity in bound $\mathrm{CO}$ spectra suggests that $\mathrm{MbCO}$ in sol-gel is in native form but modification in the spectra indicates that the potential energy surface of the protein is altered by the encapsulation. ${ }^{14}$ It has been suggested that the environment of proteins encapsulated in silica sol-gel glasses is similar to that in aqueous solution with an increased viscosity. ${ }^{+15}$ It has been noted that a large fraction of the protein surface remains in contact with the nanoscopic water surrounding the protein in the pore. ${ }^{+}$The mean pore diameters in aged wet sol-gel are comparable to the diameter of $\mathrm{Mb}$. The protein structural stability is maintained by the surrounding solvent but the dynamics of protein is strongly influenced by the dynamics of the surrounding solvent, which can be affected by the pore dimension. Time evolution of the vibrational bands of $\mathrm{CO}$ photodissociated from $\mathrm{MbCO}$ in sol-gel at $283 \mathrm{~K}$ is shown in Figure $\mathrm{l}(\mathrm{b})$. The spectral features in sol-gel are very similar to those in water: the spectral intensity initially grows due to protein rearrangement. $^{8}$ the bands shift and narrow due to conformational relaxation of the protein, ${ }^{8,10.11}$ and the fractional population of each band evolves due to interconversion of ligand within heme pocket. ${ }^{16}$ In this note we will focus on the spectral evolution of the main bands resulting from conformational relaxation of $\mathrm{Mb}$ after deligation. The spectral evolution of $B$ bands was characterized using the same method as in our previous reports. ${ }^{1011}$ Absorption features were fit to two evolving bands each of which is modeled with sum of two Gaussians. In each band, the parameters for the small Gaussian were slaved to the main Gaussian by a shift for center wavenumber. a scale factor for standard deviation, 

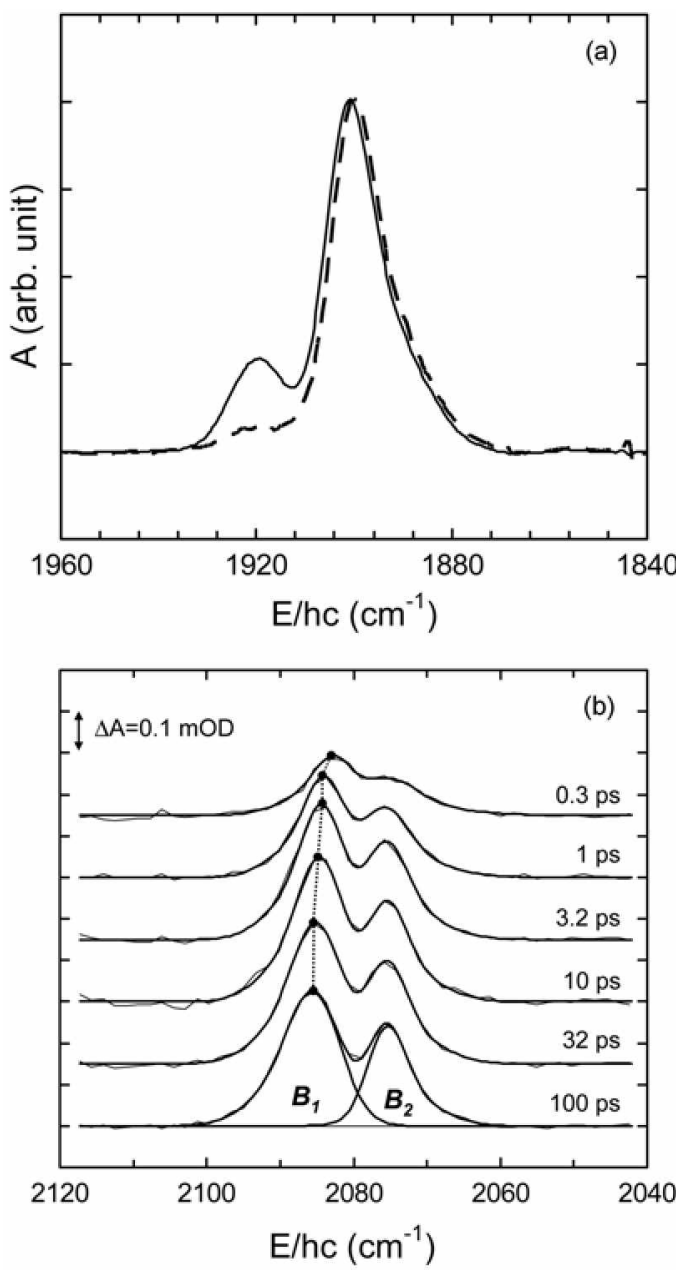

Figure 1. (a) Nornalized and background subtracted IR absorption spectra of the stretching mode of ${ }^{13} \mathrm{CO}$ bound to $\mathrm{Mb}$ in $\mathrm{D}_{2} \mathrm{O}$ (dashed line) and encapsulated in sol-gel glasses (solid line). The magnitude of $A_{1}$ band at $1920 \mathrm{~cm}^{-1}$ is about $1 / 5$ and $1 / 17$ of that of main band in sol-gel and in $\mathrm{D}_{2} \mathrm{O}$, respectively. (b) Representative time-resolved vibrational spectra of $\mathrm{C} O \mathrm{O}$ photolyzed from $\mathrm{MbC} O \mathrm{O}$ in sol-gel at $283 \mathrm{~K}$ (data: thin lines, fit: thick lines). Vibrational hot band and cubic polynomial representing background solvent change $\mathrm{e}^{10}$ are subtracted from the data for clarity. The center frequencies of $B_{1}$ bands, $M f_{1}$ s are located by filled circles and connected by dotted line to show the trend.

and another scale factor for integrated absorbance. Robust spectral parameters characterizing $B$-states were recovered by global fit of the whole spectra using these three global parameters and parameters characterizing evolution of one Gaussian for each band (see Figure 1(b)). The first and the second moments $\left(M_{1}\right.$ and $\left.M_{2}\right)$ were calculated from the fitted parameters to describe spectral evolution of $B$ bands. As can be seen in Figure 2. $B$ band narrows significantly at modestly shifting position. and $B_{1}$ band shifts toward higher energy and broadens. As compared in Figure 2, while the overall trend of the time evolution of both bands of $\mathrm{Mb}$ in sol-gel is similar to those in various solvents studied in this lab. the shift of $B_{1}$ band for the sol-gel sample is much slower than that for the aqueous saniple but faster than that in trehalose. The slower shift indicates that solvent-coupled confor-

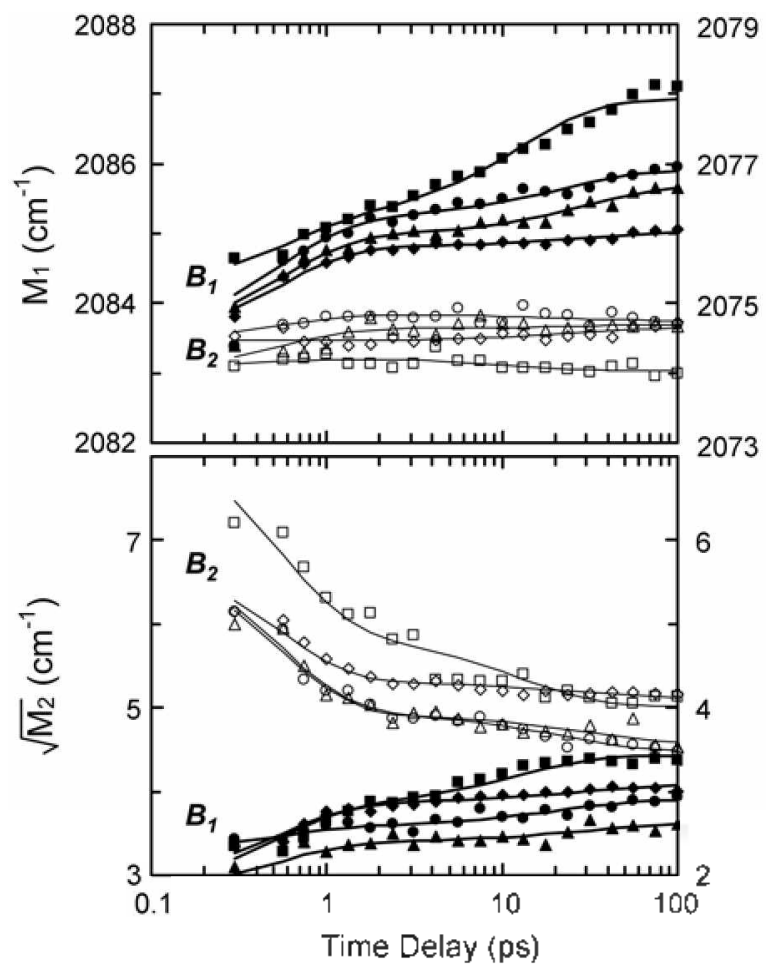

Figure 2. Time evolution of the moments $\left(h_{1}\right.$ and $\left.h_{2}\right)$ describing $B_{1}$ band (filled symbols, left ordinate) and $B_{2}$ band (open symbols, right ordinate) in $\mathrm{D}_{2} \mathrm{O}$ (squares), sol-gel (circles ), $75 \mathrm{wt} \%$ glycerol/ water mixture (triangles) and trehalose (rhombuses).

mational relaxation of $\mathrm{Mb}$ in sol-gel after deligation occurs more slowly than that in water. The total integrated area of both bands (not shown here) grows exponentially with a time constant of $1.1 \pm 0.1 \mathrm{ps}$ and is maintained a constant magnitude thereafter. It is the same as what is observed in other solvents and consistent with the suggestion that motions involved in protein arrangement constraining the orientation of the docked $\mathrm{CO}$ after photoly sis are negligibly coupled to the solvent. ${ }^{11}$

Time evolution of the first and the second moments of $B$ bands of $\mathrm{CO}$ photolyzed from $\mathrm{MbCO}$ and $\mathrm{HbCO}_{2}$ in $\mathrm{D}_{2} \mathrm{O} .75$ $\mathrm{wt} \%$ glycerol/water mixture and trehalose was described by biexponential function in the time span of $100 \mathrm{ps}$ after photolysis. "Time evolutions of both moments of the two bands for $\mathrm{Mb}$ and $\mathrm{Hb}$ in three different solvent were well reproduced by a fast global time constant for all six samples and six slow time constants shared for both moments of the two bands of each sample. The recovered fast global time constant is $0.52 \pm 0.05 \mathrm{ps}$ and the slow time constants are ranging from 13 to $32 \mathrm{ps} .^{11}$ While the magnitude decaying with the fast global time constant is about the same in all six samples. the slow time constants tend to be getting longer and their magnitude are getting smaller with increasing solvent viscosity and the presence of quaternary contact in $\mathrm{Hb}$. These results led us to suggest that the fast spectral evolution arises from local dynamics of protein interior poorly coupled with any solvent-coupled global protein dynamics and results from an intrinsic protein dynamics well reserved in heme proteins. and the magnitude of the slow 
Table 1. The fitted parameters of $\Delta \mathrm{H} f_{1}$ for $B_{1}$ band of $\mathrm{MbCO}$ at various solvents. $\Delta M_{1}$ for $B_{1}$ band in all four solvents are globally fit to a biesponential function of the fonn $a_{f}$ exp $\left(-t / \tau_{f}\right)+a_{s}$ exp $(-t /$ $\tau_{s}$ ) with a shared time constant, $t_{f}$

\begin{tabular}{ccccc}
\hline solvent & $\tau_{f}(\mathrm{ps})$ & $\tau_{s}(\mathrm{ps})$ & $a_{f}$ & $a_{s}$ \\
\hline $\mathrm{D} \approx \mathrm{O}$ & & $13 \pm 1$ & 1.1 & 1.8 \\
sol-gel & & $22 \pm 1$ & 1.9 & 0.7 \\
$75 \mathrm{wt} \% \mathrm{G} / \mathrm{W}$ & $0.52 \pm 0.05$ & $32 \pm 1$ & 1.7 & 0.7 \\
trehalose & & $28 \pm 1$ & 1.6 & 0.2 \\
\hline
\end{tabular}

spectral shift of $B_{1}$ band is a good reporter for the extent of global conformational relaxation of heme proteins after deligation. ${ }^{11}$ Here we refit time evolution of both moments of the two bands for $\mathrm{Mb}$ in four different solvents including sol-gel. The recovered parameters are consistent with those found in previous experiments. confirming our earlier suggestion. Parameters for time evolution of $B_{1}$ band are shown in Table 1. The slow time constant and its magnitude of $\Delta M_{1}$ for $B_{1}$ band of $\mathrm{MbCO}$ in sol-gel suggests that effect of spatial confinement imposed by sol-gel on $\mathrm{Mb}$ is equivalent to the effect caused by more viscous aqueous solution and the viscosity due to encapsulation in sol-gel glasses is smaller than that of $75 \mathrm{wt} \%$ glycerol/water mixture. It is consistent with a recent IR vibrational echo spectroscopy of $\mathrm{MbCO}$. suggesting that dynamics of $\mathrm{MbCO}$ encapsulated in sol-gel is the equivalent of the protein in about 20 times more viscous solution than bulk water. ${ }^{+115}$ The viscosity of $75 \mathrm{wt} \%$ glycerol/water mixture is calculated to be about 36 times more viscous than bulk water. It was estimated that in silica sol-gel glasses $\mathrm{Mb}$ is encapsulated in the pore where the protein is surrounded by less than 2-nm-thick water layer. ${ }^{2}$ While protein exterior can interact with the hydrophilic pore walls. a large fraction of the protein surface is likely solvated by nanoscopic water in the pore. The thin layer of water between a protein and pore walls of sol-gel matrix likely helps the protein with maintaining its native structure while retards its global motion coupled to solvent.

In conclusion we have confirmed our previous suggestion that picosecond spectral evolution of CO photolyzed from $\mathrm{MbCO}$ is a good reporter for solvent-coupled global conformational relaxation of $\mathrm{Mb}$ after deligation and global conformational dy'namics of $\mathrm{MbCO}$ in silica sol-gel is equivalent to that of the protein in nearly 20 times more viscous aqueous solution.

\section{Experimental Section}

Sol-gel-encapsulated sample was prepared by rapidly mixing $90 \mu \mathrm{L}$ of $6.8 \mathrm{mM} \mathrm{MbCO}$ solution and $4.5 \mu \mathrm{L}$ of buffer with $28 \mu \mathrm{L}$ of sol (molar ratio of silane precursor to water, $R$ value is 30 ), ${ }^{2,4} \mathrm{MbCO}$ was prepared by dissolving lyophilized skeletal horse $\mathrm{Mb}$ (Signa) in $50 \mathrm{mM}$ MES buffer ( $\mathrm{pH} 5.5)$. reducing the solution with $c a .2$ equivalent concentration of freshly prepared sodium dithionate (Aldrich). and then stirring the reduced $\mathrm{Mb}$ under $1 \mathrm{~atm}{ }^{13} \mathrm{CO}$ for at least 1 hour. Light scattering sources such as dust particles and denatured protein aggregates were removed by filtering samples through a $0.45 \mu \mathrm{m}$ membrane filter. The sol was prepared by combining $0.92 \mathrm{~mL}$ tetramethosy silane with $0.21 \mathrm{~mL} \mathrm{D_{2 }} \mathrm{O}$ and $0.014 \mathrm{~mL}$ of $0.04 \mathrm{~N} \mathrm{HCl}$ in $\mathrm{D}_{2} \mathrm{O}$ and then sonicating the solution for $10 \mathrm{~min}$. To slow gelation process. $\mathrm{MbCO}$ and sol was prepared in $\mathrm{pH} 5.5$ buffer instead of $\mathrm{pH}$ 7.4 phosphate. After rapidly mixing sol. $\mathrm{MbCO}$ and buffer solution for $10 \mathrm{sec}$, the mixture was immediately loaded in a sample cell with two $\mathrm{CaF}_{2}$ windows and a 77- $\mu \mathrm{m}$ Teflon spacer. The spacer was cut into two discontinuous pieces to permit exchange of methanol and buffer during soaking process in $\mathrm{pH} 7.4$ phosphate buffer. The $\mathrm{pH}$ inside sol-gel sample was neutralized after $15 \mathrm{~h}$. After neutralization, vacuum grease was applied to the edge openings of the Teflon spacer to seal it and thus prevent drying and protein oxidation.

Two identical home-built optical parametric amplifiers (OPA). pumped by a commercial Ti:sapphire amplifier. were used to generate a visible pump pulse and a mid-IR probe pulse. ${ }^{1.13}$ Pump pulse at $580 \mathrm{~nm}$ with $3 \mu \mathrm{J}$ of energy was generated by frequency doubling of a signal pulse of one OPA. Tunable mid-IR probe pulse was generated by difference frequency mixing of the signal and idler pulse of the other OPA. The polarization of the pump pulse was set at the magic angle relative to the probe pulse to recover the isotropic absorption spectrum. The broadband transmitted probe pulse was detected with a 64-elements $\mathrm{N}_{2}(l)$-cooled HgCdTe array detector. ${ }^{13}$ The array detector is mounted in the focal plane of a $320 \mathrm{~mm}$ monochromator with a 150 $l / \mathrm{nm}$ grating. resulting in a spectral resolution of $c a .1 .4 \mathrm{~cm}^{-1} /$ pixel at $2100 \mathrm{~cm}^{-1}$. The instrument response function is typically $150 \mathrm{fs}$. During data collection the sample cell was rotated sufficiently fast so that each pump pulse illuminated a fresh volume of the sample. The temperature of the rotating sample cell was maintained at $283 \pm 1 \mathrm{~K}$. The integrity and concentration of sample was checked using UV-Vis and FT-IR spectroscopy:

Acknowledgements. This work was supported by the SRC program of MOST/KOSEF (RII-2000-070-04004-0, Center for Integrated Molecular Systems) and the KRF Grant funded by the Korean Government (MOEHRD) (KRF-2005-070-C00063). We thank Mr. J. Heo for his help in sample preparation. We are grateful to the Central Laboratory at Pusan National University for our unrestricted access to the femtosecond laser system.

\section{References}

1. (a) Agmont. N.: Hoptield. . J. J. Chen. Phws. 1983. 79. 20422053. (b) Kimn. S.: Limn. M. J. Am. Chent. Soc. 2005. 127. 89088909.

2. Ellerby. L. M.: Nishida. C. R.: Nishida. F.: Yamanaka. S. A.: Dutun. B.: Valentine. J. S.; Zink. J. I. Science 1992. 255. 1113-1115.

3. Gill. I. Chem 1 Hater 2001, 13, 3404-3421.

4. Massari, A. M.: Finkelstein. I. J.; Faver, M. D. J. Am. Chem. Soc. 2006. 128. 3990-3997.

5. (a) Das. I. K.: Khan. I.: Rousseaut. D. L.: Friedman. J. M. J. Atnt Chent. Soc. 1998. 120. 10268-10269. (b) Williams. A. K.: Hupp. J. T. J. Am. Chem. Soc. 1998, 120. 4366-4371. (c) Bruno. S.; 
Bonaccio. M.: Bettati. S.: Rivetti. C.: Vappiani. C.: Abbruzzetti. S.: Mozzarelli. A. Protein Sci 2001. 10. 2401-2407. (d) Eggers. D. K.: Valentine. J. S. Protein Sci. 2001. 10. 250-261.

6. (a) Bettati. S.: Mozzarelli. A. J. Biol. Chem. 1997, 272. 3205032055 . (b) Shibavama. N. J. Hol. Biol. 1999, 285, 1383-1388. (c) Khan, I.: Shannon. C. F.: Dantsker. D.: Friedman, A. J: PerezGonzalez-de-Apodaca. I. Friedman, J. M. Biochem. 2000, 39. 16099-109. (d) Samuni. U.: Dantsker. D.: Khan. I.: Friedman. A. J.: Peterson. E.: Friedman. J. M. J. Biol. Chem. 2002. 277. 2578325790 .

7. Austin. R. H: Beeson, K. W: Eisenstein, L.: Fraulenfelder. H.: Gunsalus. I. C. Biochem. 1975. 14. 5355-5373.

8. Lim, M: Tackson. T. A.: Anfinrud. P. A. Nature Strtct Biol. 1997. 4. 209-2l4

9. Lim. M.: Jackson. T. A.: Anfinrud. P. A. J. Chent. Phys. 1995.
102. $4355-4366$

10. Kim. S.: Heo. J.: Lim. M. Bull. Korean Chent. Soc. 2005. 26. 151156.

11. Kiml. S.: Lim. M. Bull. Korean Chent. Soc. 2006. 27. 1825-1831,

12. Hamm. P.; Kaindl, R. A.: Stenger, J. Opt. Lett. 2000. 25, 17981800.

13. Kim. S.: Jin, G.; Lim, M. J. Phus. Chem. B 2004. 108, 20366 20375 .

14. McClain. B. L.: Finkelstein. I. T.: Fayer. M. D. J. Am. Chem. Soc 2004. 126. 15702-15710

15. Abbruzzetti, S.: Viappiani, C.: Bruno, S.; Mozzarelli, A. Chem Phus. Lett. 2001. $346.430-436$

16. (a) Kim. S.: Lim. M. J. Am. Chem. Soc. 2005. 127. 5786-5787. (b) Kiml. S.: Heo. T.: Lim. M. J. Am. Chem. Soc. 2006. 128. 2810 2811. 